\title{
REDUCING DISTORTION AND INTERNAL FORCES IN TRUSS STRUCTURES BY MEMBER EXCHANGES
}

\author{
(NASA-TA-101535) BEDUCIHG DISIOETICA AUD M89-16194 \\ IUTERBLL FORCES IA TRLSS STECCTUEES BY \\ LEABER EXCHAHERS (BASA) 2E F CSCI $20 \mathrm{R}$

$\begin{array}{ll}\text { G } 3 / 39 & \text { Onclas } \\ 0189728\end{array}$

W. H. Greene and R. T. Haftka

January 1989

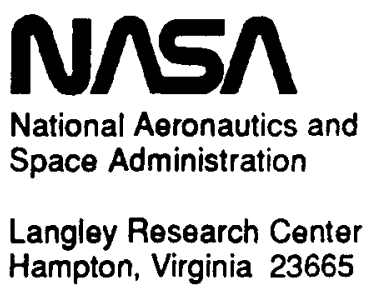




\title{
REDUCING DISTORTION AND INTERNAL FORCES IN TRUSS STRUCTURES BY MEMBER EXCHANGES
}

\author{
William H. Greene* \\ NASA Langley Research Center, Hampton, VA 23665 \\ and \\ Raphael T. Haftka $\dagger$ \\ Virginia Polytechnic Institute and State University, Blacksburg, VA 24061
}

\begin{abstract}
Inaccuracies in the length of members or diameters of joints of large truss reflector backup structures may result in unacceptably large surface distortions and member forces. However, it may be possible to accurately measure these length or diameter errors. The present work suggests that a member and joint placement strategy can markedly reduce the distortions and internal member forces. To demonstrate the potential of this approach, a member and joint exchange algorithm developed herein is applied to several 102-member and 660 -member truss reflector structures. It is shown that member and joint exchanges can simultaneously reduce the rms surface error and the rms member forces by two orders of magnitude.
\end{abstract}

* Aerospace Engineer, Structural Mechanics Division.

$\uparrow$ Christopher Kraft Professor of Aerospace and Ocean Engineering. 


\section{Nomenclature}

$A_{i} \quad=$ cross-sectional area of $\mathrm{i}$-th member

$\mathbf{B}_{d} \quad=$ weighting matrix for displacement rms, Eq. (3)

Bs = weighting matrix for member force rms, Eq. (10)

d = vector of displacements relative to the best-fit surface

$\mathrm{D} \quad=$ reflector diameter

$d_{r m s}=$ displacement rms, Eq. (3)

$d_{\text {orms }}=$ displacement rms before member exchange

$\overline{d_{r m s}}=$ nondimensional surface error parameter, Eq. (28)

$E_{i} \quad=$ Young's modulus of i-th member

f $\quad=$ vector of external forces

$f \quad=$ focal length of the ideal paraboloid

$f_{i} \quad=$ force simulating i-th member length error, Eq. (7)

$\mathrm{g}=d_{r m s}^{2}$ gradient vector, Eq. (19)

$\mathbf{H}=d_{r m s}^{2}$ Hessian matrix, Eq. (17)

$J \quad=$ objective function combining $d_{r m s}$ and $s_{r m s}$

$\mathbf{K}=$ system stiffness matrix

$l_{i} \quad=$ length of $\mathrm{i}$-th member

$\mathbf{P} \quad=$ projection matrix from the best-fit analysis, Eq. (5)

$\mathbf{R}=$ matrix of rigid body modes used in the best-fit analysis

S = member force influence coefficient matrix, Eq. (15)

s $\quad=$ vector of internal forces

$s_{r m s}=$ member-force rms, Eq. (10)

$\mathrm{s}_{\text {orm }} \mathrm{s}=$ member force rms before member exchange

$\overline{s_{r m s}}=$ nondimensional member force parameter, Eq. (29)

$\mathbf{T}=$ coordinate transformation matrix, Eq. (8)

$\mathbf{U}=$ displacement influence coefficient matrix, Eq. (14)

$\mathrm{u} \quad=$ vector of displacements

$\mathbf{w}=$ vector of top surface displacement components normal to the ideal reflector surface

$\mathbf{z}_{i}=$ adjoint load vector associated with $\mathrm{i}$-th component of $\mathrm{d}$ 
$\alpha \quad=$ vector of amplitudes obtained from solution of Eq. (4)

$\beta=$ objective function weighting parameter, Eq. (11)

$\delta \quad=$ minimum acceptable change in the objective function during a member exchange

$\lambda_{i} \quad=$ adjoint vector associated with $\mathrm{i}$-th component of $\mathbf{d}$

$\sigma_{\epsilon} \quad=$ standard deviation of the error strain in truss members

\section{Introduction}

Manufacturing errors in the length of individual members and in the size of joints cause two problems in large truss structures. First, many truss structures for space applications, such as those supporting antenna reflectors, are required to have an accurate surface shape. Errors in member length distort this surface shape (e.g. Ref. 1). Second, because most truss structures are structurally redundant, member length errors cause built-in forces in the truss members (e.g. Ref. 2). These forces can be a significant fraction of the design load for the member and can also adversely affect procedures for assembly of the truss.

A number of studies have addressed the use of actuators in the structure to reduce the surface distortion due to manufacturing errors or other sources (e.g. Refs. 3-5). One conclusion of these studies is that many actuators are needed to reduce the surface error significantly (e.g. Ref. 5). Surface errors and member forces may also be reduced, without resorting to active control, by imposing stricter manufacturing tolerances on members and joints. However, this approach increases manufacturing costs and may not be capable of achieving the desired surface accuracy for applications demanding extreme precision. This paper proposes an alternate approach to dealing with the problem of manufacturing errors in truss components.

Errors in member lengths or joint diameters due to the manufacturing process are generally assumed to be random in nature. Previous studies have assumed probability distributions for the member length errors and used statistical methods to estimate the effects on surface accuracy and member forces (e.g. Refs. 1-3). The output from these studies describes the surface error and member forces statistically.

The present paper, on the other hand, assumes that it is possible and desirable to accurately measure the member lengths and joint diameters for a given truss. Then, for a 
particular arrangement of members and joints, it is possible to predict the specific set of surface distortions and member forces of the truss.

Then the obvious question arises as to which arrangement of members and joints is best. This paper develops a method for finding arrangements which reduce the surface distortion and member forces. Several numerical studies are performed using tetrahedral trusses with 102 and 660 members to demonstrate the effectiveness of this method. In most cases the errors are generated as zero-mean random numbers with a normal distribution. In one case, the member length errors and joint diameter errors are taken from measurements of actual truss hardware. In all cases, the member exchange method finds new arrangements of members that greatly reduce the surface distortion and built-in member forces.

\section{Tetrahedral Truss Geometry}

The truss structure considered in this study is one that may be used to support a precision segmented reflector or antenna surface, and its geometry is shown in Fig. 1. The structure has a hexagonal planform and is characterized by the number of rings of members in the truss. A typical ring is shown as the shaded region on the five-ring truss in Fig. 1.

Both flat and paraboloidal truss geometries are considered. In the flat case all members are designed to have equal nominal lengths. For the paraboloidal geometry, the joint locations are obtained by first generating coordinates for a flat truss. Then the change in vertical position of the joints on the concave top surface is found directly from the equation

$$
z=\frac{r^{2}}{4 f}
$$

where $x$ and $y$ are the same as for the flat truss, $r^{2}=x^{2}+y^{2}$, and $f$ is the focal length of the paraboloid. The $x, y$, and $z$ locations for the joints on the convex bottom surface are obtained by requiring the lengths of all members in the core to be equal. In the resulting reflector geometry, the nominal lengths of of the members in both the top and bottom surfaces vary slightly from one another, with some repetition due to the hexagonal symmetry. This variation in member length significantly limits the number of members 
having a given nominal length, and hence the number of possible member exchanges. When the nembers and joints all have their nominal dimensions, the truss has this ideal geometry, and it is assumed to be stress-free. Deviations in member lengths or joint diameters from this condition cause distortions and residual forces to develop in the structure. In this study it is assumed that joint diameter errors can be directly translated into equivalent member length errors.

\section{Calculation of Surface Error and Member Forces}

The truss structure (see Fig. 1) is assumed to support a reflector surface at its upper joints. This surface is usually required to be paraboloidal or possibly flat. Due to the member length and joint diameter errors the surface will be distorted from this ideal shape. A commonly used error measure for the distorted shape is its best-fit root-mean-square (rms) deviation from the ideal shape which is defined as follows herein.

The best-fit surface is defined as the surface for which the mean-square error between the distorted truss joints and the ideal surface is minimum. In determining the best-fit surface, the ideal surface is allowed to translate along the $z$ axis and rotate about the $x$ and $y$ axes relative to the distorted surface defined by the structural joints. Translations along the $x$ and $y$ axes are not included in the best-fit analysis because they are undetermined for a flat reflector and have only a small effect for shallow, curved reflectors. The resulting best-fit displacement vector $\mathbf{d}$ is given as

$$
\mathbf{d}=\mathbf{w}-\mathbf{R} \boldsymbol{\alpha}
$$

where $\mathbf{w}$ is the vector of components of joint displacements normal to the undistorted reflector surface, $\mathbf{R}$ is a matrix of the three rigid-body modes, and $\alpha$ is a vector of amplitudes. The vector $\alpha$ is determined by minimizing the weighted rms value of $\mathbf{d}$ given as

$$
d_{r m s}^{2}=\mathbf{d}^{T} \mathbf{B}_{d} \mathbf{d}
$$

The matrix $\mathbf{B}_{d}$ is a positive semidefinite weighting matrix (an identity matrix divided by the number of components of $\mathbf{d}$ in the examples herein). The vector $\alpha$ which minimizes 
$d_{r m s}^{2}$ is found by substituting Eq. (2) into Eq. (3) and differentiating with respect to $\alpha$ to get the solution to the normal equations,

$$
\boldsymbol{\alpha}=\left(\mathbf{R}^{T} \mathbf{B}_{d} \mathbf{R}\right)^{-1} \mathbf{R}^{T} \mathbf{B}_{d} \mathbf{w}
$$

so that

$$
\mathbf{d}=\left[\mathbf{I}-\mathbf{R}\left(\mathbf{R}^{T} \mathbf{B}_{d} \mathbf{R}\right)^{-1} \mathbf{R}^{T} \mathbf{B}_{d}\right] \mathbf{w}=\mathbf{P} \mathbf{w}
$$

where $\mathbf{P}$ is called the projection matrix. The finite element model of the truss incorporates statically determinate rigid body restraints to make the stiffness matrix $\mathbf{K}$ nonsingular. The finite element analysis, based on small-deformation linear analysis, solves the system

$$
\mathbf{K u}=\mathbf{f}
$$

where $\mathbf{u}$ is the restrained motion due to member length errors and $\mathbf{f}$ is the set of equivalent member forces that simulate the effect of member length errors. The contribution of the $\mathrm{i}$-th member of the truss is a pair of forces colinear with the member of magnitude

$$
f_{i}=\frac{E_{i} A_{i}}{l_{i}} e_{i}
$$

where $E_{i}, A_{i}, l_{i}$, and $e_{i}$ are the member Young's modulus, cross-sectional area, length and length error, respectively.

The vector of upper surface joint displacements normal to the ideal surface is obtained by substituting the solution to Eq. (6) into

$$
\mathbf{d}=\mathbf{P T u}
$$

The matrix $\mathbf{T}$ extracts from the components of $\mathbf{u}$ at each joint in the computational coordinate system a displacement normal to the undistorted surface given as

$$
w_{i}=\mathbf{t}_{i} \mathbf{u}
$$

where $w_{i}$ is the normal displacement, and $\mathbf{u}$ is the vector of displacements in the computational coordinate system. The components of the vector $t_{i}$, the $i$-th row of $\mathbf{T}$, are all zero except for the three direction cosines of the surface normal for the $\mathrm{i}$-th joint at the 
appropriate locations in the row. Then $d_{r m s}$ for the distorted surface can be calculated using Eqs. (8) and (3).

The member force vector, $\mathbf{s}$, is also of interest and can be calculated using the solution vector, $u$. For the member forces a measure of magnitude is $s_{r m s}$ defined as

$$
s_{r m s}^{2}=\mathbf{s}^{T} \mathbf{B}_{s} \mathbf{s}
$$

where $B_{s}$ is a positive semidefinite matrix (an identity matrix divided by the number of members in the examples herein).

\section{Member Exchange}

Once the members of the truss and its joints are manufactured, an initial configuration corresponding to a particular placement of member and joints is selected. The initial configuration is defined by a set of member and joint errors with corresponding normal displacement and member force measures, $d_{\text {orms }}$ and $s_{\text {orms }}$, respectively. A compromise objective that combines distortion and member forces is selected as

$$
J=\beta\left(\frac{d_{r m s}}{d_{\text {orms }}}\right)^{2}+(1-\beta)\left(\frac{s_{r m s}}{s_{o r m s}}\right)^{2}
$$

The value of $\beta$ can be selected to emphasize either low distortion or low forces or some compromise between the two requirements.

The objective function is then reduced by a binary or triple member exchange algorithm. The binary member exchange algorithm takes the following form

1. Select a minimum acceptable objective function improvement fraction $\delta$ (per iteration), to invoke a member exchange.

2. Start with the first member.

3. Calculate the change $\Delta J$ obtained by exchanging the current member with other members (starting with the first) until an exchange is found that results in $(-\Delta J / J)>$ $\delta$.

4. Execute the exchange (if any).

5. If not at the last member, move to the next member and go back to step 3 . 
6. If at the last member, check whether the total number of iterations is exceeded; if yes, stop.

7. If the total number of iterations is not exceeded, reduce $\delta$ and go to step 2 .

The binary exchange strategy is not guaranteed to lead to the minimum. Ilowever, increasing the number of members involved in a single exchange improves the chances of locating the minimum. When all members can be exchanged at each step the minimum is guaranteed to be found in a single iteration, but the number of exchanges that is considered in that single iteration is the factorial of the number of members. For illustration the Appendix presents a simple system where no binary exchange can improve the objective function but a triple exchange can reduce it to almost zero. Accordingly, a more expensive triple exchange was also used for some examples. The triple exchange algorithm follows the same procedure as the binary exchange, except that in step 3 above, the current member is matched with all combinations of any two other members. Of course, a triple exchange algorithm still does not guarantee that the absolute minimum of $J$ will be found.

\section{Efficient Reanalysis}

For trusses with several hundred members, the member exchange algorithm requires the evaluation of hundreds of thousands to millions of configurations. Therefore, a technique for the inexpensive evaluation of the effect of an exchange is required. Because the force vector $\mathbf{f}$ is linearly proportional to the member length and joint errors, and $\mathbf{d}$ is a linear function of $\mathbf{f}$,

$$
\mathbf{d}=\mathbf{U}_{l} \mathbf{e}_{l}+\mathbf{U}_{r} \mathbf{e}_{r}
$$

where $\mathbf{e}_{l}$ and $\mathbf{e}_{r}$ are vectors of member length and joint diameters errors, respectively, and $\mathbf{U}_{l}$ and $\mathbf{U}_{r}$ are matrices of influence coefficients. Similarly, the vector of member forces can be written as

$$
\mathbf{S}=\mathbf{S}_{l} \mathbf{e}_{l}+\mathbf{S}_{r} \mathbf{e}_{r}
$$

where $\mathbf{S}_{l}$ and $\mathbf{S}_{r}$ are matrices of influence coefficients. The error vectors $\mathbf{e}_{l}$ and $\mathbf{e}_{\boldsymbol{r}}$ can be concatenated into a vector $\mathbf{e}$, and the matrices of influence coefficients $\mathbf{U}_{l}$ and $\mathbf{U}_{r}$ and $\mathbf{S}_{l}$ 
and $\mathbf{S}_{r}$ similarly concatenated into matrices $\mathbf{U}$ and $\mathbf{S}$, respectively. Then Eqs. (12) and (13) may be written as

$$
\mathbf{d}=\mathbf{U e}
$$

and

$$
\mathbf{s}=\mathbf{S e}
$$

Now using Eqs. (3) and (14) the mean-squared error can be written as

$$
d_{r m s}^{2}=\mathbf{e}^{T} \mathbf{H e}
$$

where

$$
\mathbf{H}=\mathbf{U}^{T} \mathbf{B}_{d} \mathbf{U}
$$

so that

$$
\Delta d_{r m s}^{2}=\mathbf{g}^{T} \Delta \mathbf{e}+\Delta \mathbf{e}^{T} \mathbf{H} \Delta \mathbf{e}
$$

where $\Delta \mathbf{e}$ denotes the change in e due to member exchange, and where $\mathbf{g}$, the gradient of $d_{r m s}^{2}$ is

$$
\mathbf{g}=2 \mathbf{H e}
$$

Note that in spite of its similarity to a quadratic approximation, Eq. (18) is exact, because $d_{r m}^{2}$ is a quadratic function. When members $i$ and $j$ are exchanged Eq. (18) gives

$$
\Delta d_{r m s}^{2}=\left(g_{i}-g_{j}\right)\left(e_{j}-e_{i}\right)+\left(e_{j}-e_{i}\right)^{2}\left(h_{i i}+h_{j j}-2 h_{i j}\right)
$$

Also, after an exchange the gradient vector $g$ has to be updated by

$$
\Delta \mathbf{g}=\mathbf{H} \Delta \mathbf{e}
$$

In a single iteration Eq. (20) is evaluated a large number of times (up to $m(m-1)$ times, where $m$ is the number of members). The more expensive Eq. (21) is executed only when an exchange takes place (up to $m$ times). Note, however, that for binary exchanges the vector $\Delta \mathbf{e}$ has only two nonzero components so that $\mathrm{Eq} .(21)$ is inexpensive to evaluate. The effect of member exchanges on $s_{r m}$, are calculated in a similar way. 
For triple exchanges with members $i, j, k$ permuted to $k, i, j$, we have

$$
\begin{aligned}
\Delta d_{r m s}^{2}= & g_{i}\left(e_{k}-e_{i}\right)+g_{j}\left(e_{i}-e_{j}\right)+g_{k}\left(e_{j}-e_{k}\right) \\
& +h_{i i}\left(e_{k}-e_{i}\right)^{2}+h_{j j}\left(e_{i}-e_{j}\right)^{2}+h_{k k}\left(e_{j}-e_{k}\right)^{2} \\
& +2 h_{i j}\left(e_{k}-e_{i}\right)\left(e_{i}-e_{j}\right)+2 h_{j k}\left(e_{i}-e_{j}\right)\left(e_{j}-e_{k}\right) \\
& +2 h_{i k}\left(e_{j}-e_{k}\right)\left(e_{k}-e_{i}\right)
\end{aligned}
$$

\section{Calculation of Influence Coefficient Matrices}

Two techniques are described for calculating the matrices of influence coefficients $U$ and $\mathbf{S}$. The first assumes that both the distortions, $\mathbf{d}$, and member forces $\mathbf{s}$ are of interest and therefore both $\mathbf{U}$ and $\mathbf{S}$ are required. This technique calculates $\mathbf{U}$ and $\mathbf{S}$ directly by calculating $\mathbf{d}$ and $\mathbf{s}$ for a unit error in each member. The second technique is based on an adjoint variable approach and is substantially cheaper than the direct approach when only $\mathbf{U}$ is required.

\section{Direct Method}

From Eq. (12) it can be seen that the $i$-th column in $\mathbf{U}_{l}$ is just the vector $\mathbf{d}$ when a unit error is introduced in the $\mathrm{i}$-th member only. This vector can be calculated using Eqs. (6), (7) and (8). Each column of the matrix $\mathbf{S}_{l}$ is obtained by calculating the member forces associated with this displacement solution. The matrices of influence coefficients $\mathbf{U}_{r}$ and $\mathbf{S}_{r}$ can then be formed as linear combinations of the columns in $\mathbf{U}_{l}$ and $\mathbf{S}_{l}$. It should be noted that this approach requires the solution of the system equations for each member in the truss. For the five- ring truss example, 660 solutions are required. However, the stiffness matrix $\mathbf{K}$ needs to be factored only once because the effect of the dimensional errors affects only the load vector. 


\section{Adjoint Variable Method}

A substantial savings in computational effort can be achieved when only surface distortions are a concern, and therefore only $\mathbf{U}$ is required. Since the number of components in $\mathbf{d}$ is typically much smaller than the number of members in the truss, a dummy load or adjoint approach is the preferred method for calculating $U$. From Eq. (8) the i-th component of $d$ may be written as

$$
d_{i}=\mathbf{b}_{i}^{T} \mathbf{d}=\mathbf{b}_{i}^{T} \mathbf{P} \mathbf{T} \mathbf{u}
$$

where $b_{i}$ is a Boolean vector with zeroes everywhere except for a one in the $i$-th position. The adjoint approach is employed by noting that the $\mathrm{i}$-th row of $\mathrm{U}_{l}$ is the vector of derivatives of $d_{i}$ with respect to the member length errors. Using the adjoint approach (e.g. Ref. 6) we apply an adjoint load $\mathbf{z}_{i}$

$$
\mathbf{z}_{i}=\left(\frac{\partial d_{i}}{\partial \mathbf{u}}\right)^{T}=\mathbf{T}^{T} \mathbf{P} \mathbf{b}_{i}
$$

to the truss and solve for the adjoint field $\boldsymbol{\lambda}_{i}$

$$
\mathbf{K} \boldsymbol{\lambda}_{i}=\mathbf{z}_{i}
$$

This equation needs to be solved for each component of $d$. For the five ring case, 91 solutions are required, as compared to 660 solutions with the direct method. The derivative of $d_{i}$ with respect to $e_{l_{j}}$ is then found as

$$
\frac{\partial d_{i}}{\partial e_{l_{j}}}=\lambda_{i}^{T} \frac{\partial \mathbf{f}}{\partial e_{l_{j}}}
$$

The right-hand side of Eq. (26) is the scalar product of the adjoint field by the force associated with a unit error in the $j$-th member which is also equal to the elongation of the $\mathrm{j}$-th member due to the adjoint load times the force in that member due to a unit length error. This may be written as (see Eq. (7))

$$
\frac{\partial d_{i}}{\partial e_{l_{j}}}=e_{\lambda_{i}} \frac{E_{j} A_{j}}{l_{j}}=s_{\lambda_{i j}}
$$

where $e_{\lambda_{i j}}$ is the elongation of the $\mathrm{j}$-th member due to $\lambda_{i}$ and $s_{\lambda_{i}}$ is the force in the $\mathrm{j}$-th member due to $\lambda_{i}$. Equation (27) implies that the $\mathrm{i}$-th row of $U$ can be calculated by applying the load $\mathbf{z}_{i}$ of Eq. (24) and calculating the resulting member forces. 


\section{Numerical Studies}

Several numerical studies have been performed to assess the potential for improvements in surface accuracy and member forces in tetrahedral trusses. Two- and five-ring flat trusses are considered with all members nominally the same length. A five-ring curved truss is also studied to assess the effectiveness of member exchanges for this case. Finally a study was performed using actual measured properties for a two-ring truss being built at NASA-Langley for an assembly experiment. In all cases all members have the same cross-sectional area and material properties.

Except for the assembly-experiment truss, the member length errors are produced using a numerical routine for generating random numbers having a normal distribution. In these cases it is convenient to generalize the results by defining two nondimensional parameters that were also used in Ref. 2. The first is a surface error parameter defined as

$$
\overline{d_{r m s}}=\frac{d_{r m s}}{D \sigma_{\epsilon}}
$$

where $D$ is the reflector diameter and $\sigma_{\epsilon}$ is the standard deviation of the error strain in the members. The second is a member force parameter defined as

$$
\overline{s_{r m s}}=\frac{s_{r m s}}{E A \sigma_{\epsilon}}
$$

where $E A$ is the member axial stiffness.

\section{Two-Ring Reflector Example}

A flat, two-ring tetrahedral reflector truss was used as the first example. All 102 members in the truss have the same nominal length so that all of them can be exchanged. The member errors were generated as a set of zero-mean random numbers having a normal distribution, and the joint errors were assumed to be zero. After some numerical experiments, an initial value of the minimum acceptable improvement per exchange, $\delta$, was selected as one percent and this value was reduced by a factor of three at each iteration.

The iteration history of $\overline{d_{r m s}}$ and $\overline{s_{r m s}}$ is shown in Table 1 for binary exchanges and in Table 2 for binary exchanges followed by triple exchanges. The strategy of applying 
binary exchanges for each iteration followed by triple exchanges for the same iteration was used to reduce the computational expense of triple exchanges. It is seen that the use of triple exchanges improves the results. For example, for $\beta=1$ the distortion rms is reduced by a factor of 146 using binary exchanges and by a factor of 229 using also triple exchanges. The corresponding numbers for the force rms $(\beta=1)$ are 237 and 333 . Use of the compromise objective function $(\beta=0.8)$ results in a reduction of $\overline{d_{r m s}}$ by a factor of 85 while simultaneously $\overline{s_{r m s}}$ is reduced by a factor of 44 .

Convergence is achieved in six or less iterations, and for the first iteration almost all members are exchanged while in the last iteration typically only one or two exchanges are made (even though the minimum acceptable improvement is greatly reduced).

\section{Five-Ring Reflector Example}

A flat version of the 660 -member five-ring reflector truss shown in Figure 1 was used as a second, larger example. All the members in the truss are of the same length so that in principle all of them could be exchanged. However, to simulate structures having different member lengths, in one case the members were divided into three groups: 240 in the upper surface, 195 in the lower surface, and 225 core elements. The first case considered, allowed any member to be exchanged with any other, and the second case allowed only members within each of the three groups to be exchanged.

A first set of nember length errors was generated using the same procedure as for the two-ring case. For this example, only surface accuracy was considered $(\beta=1.0)$. Because of the increased number of members only binary exchanges were considered. Again, $\delta$ was chosen as 0.01 initially, and was reduced by a factor of three at each iteration.

The iteration history of $\overline{d_{r m s}}$ is shown in Table 3. Again, convergence is achieved in

six iterations and $\overline{d_{r m s}}$ is reduced by a factor of 684 . Another indication of convergence was that for the first iteration almost all members were exchanged, whereas for the last iteration only 11 out of 660 members were exchanged. This took place even though in the first iteration each exchange required at least a 1 percent improvement in $d_{r m s}^{2}$, while in the last iteration the required improvement was $3^{6}=729$ times smaller. 
Table 1. Iteration history for a 2-ring, flat, 102-member truss (random length errors) using binary exchanges

\begin{tabular}{cccccccc}
\hline \hline Iteration & \multicolumn{2}{c}{$\beta=1.0$} & \multicolumn{2}{c}{$\beta=0.8$} & \multicolumn{2}{c}{$\beta=0.0$} \\
& $\overline{d_{r m s}}$ & $\overline{s_{r m s}}$ & $\bar{d}_{r m s}$ & $\overline{s_{r m s}}$ & $\overline{d_{r m s}}$ & $\overline{s_{r m s}}$ \\
\hline 0 & 0.245 & 7.55 & 0.245 & 7.55 & 0.245 & 7.55 \\
1 & 0.0314 & 11.58 & 0.0315 & 1.94 & 0.287 & 0.237 \\
2 & $0.410 \times 10^{-2}$ & 10.12 & $0.982 \times 10^{-2}$ & 0.748 & 0.388 & 0.0415 \\
3 & $0.199 \times 10^{-2}$ & 10.13 & $0.520 \times 10^{-2}$ & 0.424 & 0.310 & 0.0331 \\
4 & $0.168 \times 10^{-2}$ & 10.12 & $0.400 \times 10^{-2}$ & 0.337 & 0.403 & 0.0320 \\
5 & $0.168 \times 10^{-2}$ & 10.12 & $0.262 \times 10^{-2}$ & 0.275 & 0.408 & 0.0318 \\
6 & $0.168 \times 10^{-2}$ & 10.12 & $0.264 \times 10^{-2}$ & 0.255 & 0.408 & 0.0318 \\
7 & $0.168 \times 10^{-2}$ & 10.12 & $0.263 \times 10^{-2}$ & 0.255 & 0.420 & 0.0318 \\
\hline \hline
\end{tabular}

Table 2. Iteration history for a 2-ring, flat, 102-member truss (random length errors) using binary and triple exchanges

\begin{tabular}{ccccccc}
\hline \hline Iteration & \multicolumn{2}{c}{$\beta=1.0$} & \multicolumn{2}{c}{$\beta=0.8$} & \multicolumn{2}{c}{$\beta=0.0$} \\
& $\overline{d_{r m s}}$ & $\overline{s_{r m s}}$ & $\overline{d_{r m s}}$ & $\overline{s_{r m s}}$ & $\overline{d_{r m s}}$ & $\overline{s_{r m s}}$ \\
\hline 0 & 0.245 & 7.55 & 0.245 & 7.55 & 0.245 & 7.55 \\
1 & $0.261 \times 10^{-2}$ & 10.96 & $0.484 \times 10^{-2}$ & 0.298 & 0.308 & 0.0341 \\
2 & $0.129 \times 10^{-2}$ & 10.96 & $0.293 \times 10^{-2}$ & 0.174 & 0.324 & 0.0253 \\
3 & $0.111 \times 10^{-2}$ & 10.95 & $0.287 \times 10^{-2}$ & 0.171 & 0.312 & 0.0253 \\
4 & $0.107 \times 10^{-2}$ & 10.95 & $0.287 \times 10^{-2}$ & 0.171 & 0.278 & 0.0239 \\
5 & $0.107 \times 10^{-2}$ & 10.95 & $0.287 \times 10^{-2}$ & 0.171 & 0.315 & 0.0227 \\
6 & $0.107 \times 10^{-2}$ & 10.95 & $0.287 \times 10^{-2}$ & 0.171 & 0.316 & 0.0227 \\
\hline \hline
\end{tabular}

Next, the case of exchanges limited to groups of members was considered. Seven iterations were applied to members in the upper surface, followed by seven iterations to members in the lower surface, and finally seven iterations to core elements. The results shown in Table 4 indicate slower convergence than in the case of unlimited exchanges. 
Table 3. Iteration history of $\overline{d_{r m s}}$ for unlimited member exchanges, five-ring, flat, 660 member truss

\begin{tabular}{cc}
\hline \hline Iteration & $\overline{d_{r m s}}$ \\
\hline 0 & 0.234 \\
1 & $0.317 \times 10^{-2}$ \\
2 & $0.822 \times 10^{-3}$ \\
3 & $0.475 \times 10^{-3}$ \\
4 & $0.358 \times 10^{-3}$ \\
5 & $0.343 \times 10^{-3}$ \\
6 & $0.342 \times 10^{-3}$ \\
7 & $0.342 \times 10^{-3}$ \\
\hline \hline
\end{tabular}

Also, the total improvement was a factor of 304 . It should be noted that exchanges in core elements were most effective.

Better convergence was obtained when single iterations were performed successively on each group. The results, summarized in Table 5, show an improvement factor of 324 . The effect of changing the order of the groups was also investigated and found to be minimal.

To check the sensitivity of the results to the set of member errors, another set of errors was generated. For this second set the initial value of $\overline{d_{r m} s}$ was 0.316 . The final values were $\overline{d_{r m s}}=0.216 \times 10^{-3}$ with unlimited member exchanges (a factor of 1460 ), and $\overline{d_{r m s}}=0.815 \times 10^{-3}$ with exchanges limited to each group (a factor of 388 improvement) These values are 37 percent lower and 13 percent higher, respectively, than the final values for the first set of errors.

\section{Five-Ring Curved Reflector Example}

This example is similar to the five-ring example above except that the truss is paraboloidal (as seen in Figure 1) with an $f / D$ of the reflector defined to be 1.0. The main consequence of this geometry is that members of many different lengths are now present in the truss which limits the opportunities for exchanges. As mentioned above, the geometry is generated such that the core member lengths are all equal. Thus, the 225 core elements 
Table 4. Iteration history of $\overline{d_{r m s}}$ for exchanges limited to groups for a five-ring, flat, 660 -member truss. Iterations completed in one group before the next group was started.

\begin{tabular}{cccc}
\hline \hline Iteration & Upper surface & Lower surface & Core elements \\
\hline 0 & 0.234 & $0.428 \times 10^{-1}$ & $0.160 \times 10^{-1}$ \\
1 & $0.564 \times 10^{-1}$ & $0.277 \times 10^{-1}$ & $0.348 \times 10^{-2}$ \\
2 & $0.502 \times 10^{-1}$ & $0.223 \times 10^{-1}$ & $0.162 \times 10^{-2}$ \\
3 & $0.468 \times 10^{-1}$ & $0.197 \times 10^{-1}$ & $0.106 \times 10^{-2}$ \\
4 & $0.449 \times 10^{-1}$ & $0.184 \times 10^{-1}$ & $0.905 \times 10^{-3}$ \\
5 & $0.438 \times 10^{-1}$ & $0.176 \times 10^{-1}$ & $0.856 \times 10^{-3}$ \\
6 & $0.432 \times 10^{-1}$ & $0.168 \times 10^{-1}$ & $0.802 \times 10^{-3}$ \\
7 & $0.428 \times 10^{-1}$ & $0.160 \times 10^{-1}$ & $0.770 \times 10^{-3}$ \\
\hline \hline
\end{tabular}

Table 5. Iteration history of $\overline{d_{r m s}}$ for exchanges limited to groups for a five-ring, flat, 660 -member truss. One iteration per group at time.

\begin{tabular}{cccc}
\hline \hline Iteration & Upper surface & Lower surface & Core elements \\
\hline 0 & 0.234 & 0.234 & 0.234 \\
1 & $0.564 \times 10^{-1}$ & $0.381 \times 10^{-1}$ & $0.697 \times 10^{-2}$ \\
2 & $0.568 \times 10^{-2}$ & $0.431 \times 10^{-2}$ & $0.213 \times 10^{-2}$ \\
3 & $0.180 \times 10^{-2}$ & $0.162 \times 10^{-2}$ & $0.108 \times 10^{-2}$ \\
4 & $0.101 \times 10^{-2}$ & $0.957 \times 10^{-3}$ & $0.850 \times 10^{-3}$ \\
5 & $0.797 \times 10^{-3}$ & $0.793 \times 10^{-3}$ & $0.777 \times 10^{-3}$ \\
6 & $0.771 \times 10^{-3}$ & $0.765 \times 10^{-3}$ & $0.744 \times 10^{-3}$ \\
7 & $0.732 \times 10^{-3}$ & $0.724 \times 10^{-3}$ & $0.723 \times 10^{-3}$ \\
\hline \hline
\end{tabular}

plus 48 elements in the faces all have the same nominal lengths and can potentially be exchanged with each other. The remaining 387 members are divided into 22 groups, seven of which have only 6 members.

The member length errors were generated as zero-mean random numbers having a normal distribution. The exchange algorithm was then run with binary exchanges restricted 
to members within each group. The objective function was chosen with $\beta=1.0$, so that only surface distortion was considered. The resulting values of $\overline{d_{r m s}}$ for each of the seven iterations are shown in Table 6. Despite the constraints on allowed exchanges, the surface accuracy, as measured by $\overline{d_{r m s}}$, has been improved by a factor of 557 .

Table 6. Iteration history of $\overline{d_{r m s}}$ for the five-ring, curved, 660 -member truss.

\begin{tabular}{cc}
\hline Iteration & $\overline{d_{r m s}}$ \\
\hline 0 & .334 \\
1 & $.164 \times 10^{-1}$ \\
2 & $.371 \times 10^{-2}$ \\
3 & $.164 \times 10^{-3}$ \\
4 & $.102 \times 10^{-3}$ \\
5 & $.779 \times 10^{-3}$ \\
6 & $.646 \times 10^{-3}$ \\
7 & $.600 \times 10^{-3}$ \\
\hline \hline
\end{tabular}

\section{Two-Ring Assembly-Experiment Truss Example}

A precision, two-ring, tetrahedral truss structure (described in reference 7) is being built at NASA-Langley for use in an experiment on robotic assembly techniques for truss structures. The geometry is nominally flat. The members are constructed from $2 \mathrm{~m}$ graphite-epoxy tubes with aluminum end fittings designed to facilitate the automated assembly process. During the assembly process, the member is connected to a joint assembly constructed from a 2.25 inch diameter (nominal), machined aluminum ball with attachments to accommodate the member end fittings.

Manufacturing errors arise from several sources. First, errors in the joint-balls occur in the machining process. Measurements of these balls showed them to be highly symmetric but with significant differences in the diameter from ball to ball. Figure 2a shows a histogram of the measured diameter errors for the 31 balls used in the truss. Errors in the 
members arise in the fabrication process and in machining operations on the member-toball attachment fittings. Prior to this study a simple exchange procedure was used to match two ball-attachment fittings with each tube with the goal of producing a set of members with nearly uniform lengths. Then this combination of tube and two ball-attachment fittings was considered as the "member" with an associated length error for use in this member exchange study. Figure $2 b$ shows a histogram of the measured distribution of length errors for the 102 members used in the truss.

First, the joint and member errors were arbitrarily assigned in the finite element model of the truss. The analysis of this configuration produced a $d_{r m s}=.0023$ inches and an $s_{r m s}=25.1 \mathrm{lbs}$ with a maximum member force of $76.7 \mathrm{lbs}$. From Figures $2 \mathrm{a}$ and $2 \mathrm{~b}$ it can be seen that the value of $d_{r m}$ is of the same order as the errors in the individual joint balls and members.

The built-in forces in the members are of particular concern in the assembly process because they translate directly to the force required by the assembler to insert the member into the structure. Accordingly, a value of $\beta=.2$ was used in the exchange algorithm to emphasize the reduction of $s_{r m s}$ over improvement in surface accuracy. Because all members in the truss have the same nominal length, any two members are candidates for an exchange. The iteration history during the binary exchange process is shown in Table 7. The final result of $d_{r m s}=0.00011$ inches and $s_{r m s}=0.097$ lbs indicates a substantial reduction in surface distortion and built-in member forces. These improvements in member force and shape accuracy have not yet been duplicated in the experiment, because of difficulty in matching analytical and experimental results.

\section{Concluding Remarks}

Member length or joint diameter errors in truss structures can cause significant shape errors or built-in forces in the members. The shape errors are of particular concern when the truss structure is being used as a support for a precision electromagnetic reflector.

A method has been proposed which finds arrangements of members and joints which dramatically reduce both surface errors and built-in member forces. The method uses a 
Table 7. Iteration history for the two-ring, flat, assembly-experiment truss using binary exchanges.

\begin{tabular}{ccccc}
\hline \hline Iteration & \multicolumn{2}{c}{$d_{r m s}$ (inches) } & \multicolumn{2}{c}{$s_{r m s}(\mathrm{lbs})$} \\
& Members & Joints & Members & Joints \\
\hline 0 & $0.231 \times 10^{-2}$ & $0.231 \times 10^{-2}$ & 25.1 & 25.1 \\
1 & $0.874 \times 10^{-3}$ & $0.645 \times 10^{-3}$ & 1.47 & 1.61 \\
2 & $0.229 \times 10^{-3}$ & $0.216 \times 10^{-3}$ & 0.767 & 0.594 \\
3 & $0.154 \times 10^{-3}$ & $0.148 \times 10^{-3}$ & 0.235 & 0.306 \\
4 & $0.137 \times 10^{-3}$ & $0.137 \times 10^{-3}$ & 0.116 & 0.116 \\
5 & $0.115 \times 10^{-3}$ & $0.115 \times 10^{-3}$ & 0.166 & 0.166 \\
6 & $0.112 \times 10^{-3}$ & $0.112 \times 10^{-3}$ & 0.094 & 0.094 \\
7 & $0.111 \times 10^{-3}$ & $0.111 \times 10^{-3}$ & 0.097 & 0.097 \\
\hline \hline
\end{tabular}

heuristic algorithm to select pairs or triples of members for exchanges. Because many evaluations of the effect of potential exchanges on distortions and member forces are required, efficient reanalysis techniques based on matrices of influence coefficients of the response quantities are used. Both a direct approach and a more computationally efficient adjoint approach are used for evaluating the matrices of influence coefficients.

The member exchange method is demonstrated by application to several two- and fivering, flat and curved tetrahedral truss reflector structures. Sets of member length errors were generated as zero-mean random numbers having a normal distribution. For every example, the exchange algorithm was able to reduce surface errors and/or member forces by at least $t$ wo orders of magnitude and in some cases three orders of magnitude. The curved reflector geometry results in truss members with many different nominal lengths. This significantly limits the candidate members for exchange with a given member. However, in a five-ring curved reflector example it was still possible to reduce surface distortion by more than two orders of magnitude. A two-ring flat truss example using measured data from available truss hardware was also considered. In this case also, the exchange algorithm was able to substantially reduce computed surface distortions and member forces. 


\section{Acknowledgment}

This work was supported in part by NASA grant NAG-1-224.

\section{Appendix - Benefit of triple exchanges}

The need for triple exchanges may be demonstrated for a simple algebraic problem where the matrix $\mathbf{H}$ of Eq. (21) is given as

$$
\mathbf{H}=\left[\begin{array}{ccc}
2 & 4 & -3 \\
4 & 10 & -4 \\
-3 & -4 & 6.6
\end{array}\right]
$$

and the error vector $\mathbf{e}$ is $\left\{\begin{array}{lll}-2 & 2 & 7\end{array}\right\}^{T}$ so that

$$
d_{r m s}^{2}=\mathrm{e}^{T} \mathrm{He}=311.4
$$

It is easy to check that all binary permutations of e increase $d_{r m}$, while the triple permutation $\mathbf{e}=\left\{\begin{array}{lll}7 & -2 & 2\end{array}\right\}^{T}$ yields $d_{r m s}=0.4$. 


\section{References}

1. Hedgepeth, J.M., "Influence of Fabrication Tolerances on the Surface Accuracy of Large Antenna Structures," AIA A Journal, Vol. 20, May 1982, pp. 680-686.

2. Greene, W.H., "Effect of Random Member Length Errors on the Accuracy of Internal Loads of Truss Antennas," Journal of Spacecraft and Rockets, Vol. 22, No. 5, 1985, pp. 554-559.

3. Burdisso, R.A. and Haftka, R.T., "Optimal Locations of Actuators for Correcting Distortions Due to Manufacturing Errors in Large Truss Structures," 6th VPI\&SU/AIAA Symposium on Dynamics and Control of Large Structures, Blacksburg, Virginia, June 1987.

4. Edberg, D.L., "Control of Flexible Structures by Applied Thermal Gradients," AIAA Journal, Vol. 25, 1987, pp. 877-883.

5. Haftka, R.T. and Adelman, H.M., "Selection of Actuator Locations for Static Shape Control of Large Space Structures by Heuristic Integer Programming," Computers and Structures, Vol. 20, No. 1-3, 1985, pp. 575-585.

6. Haftka, R.T. and Kamat, M.P., Elements of Structural Optimization, Martinus Nijhoff, 1985, p. 156.

7. Rhodes, Marvin D. and Wu, K. Chauncey, "Experimental Measurement of Fabrication Errors in Components of a Regular Tetrahedral Truss." NASA TM-101555, February, 1989. 


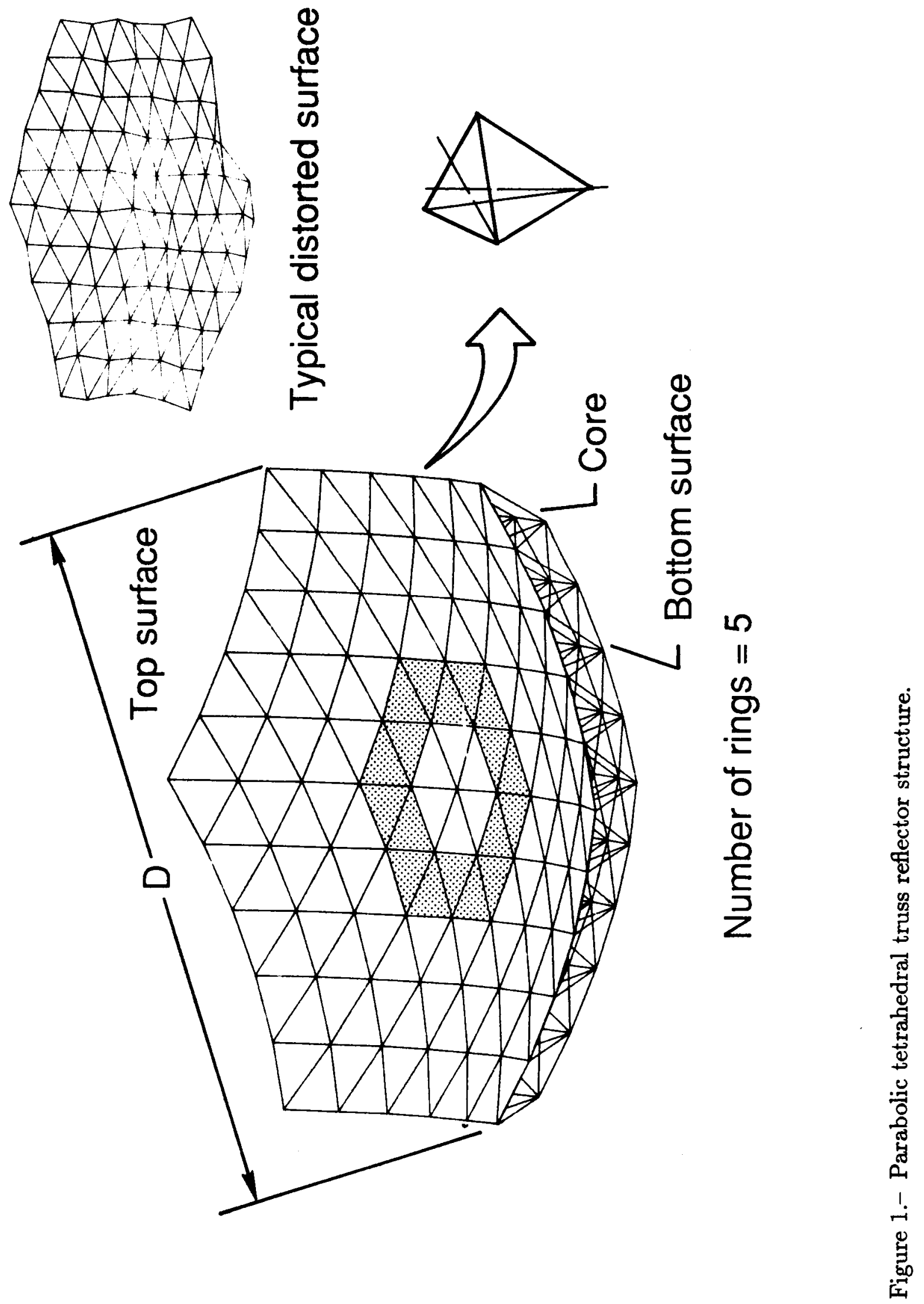



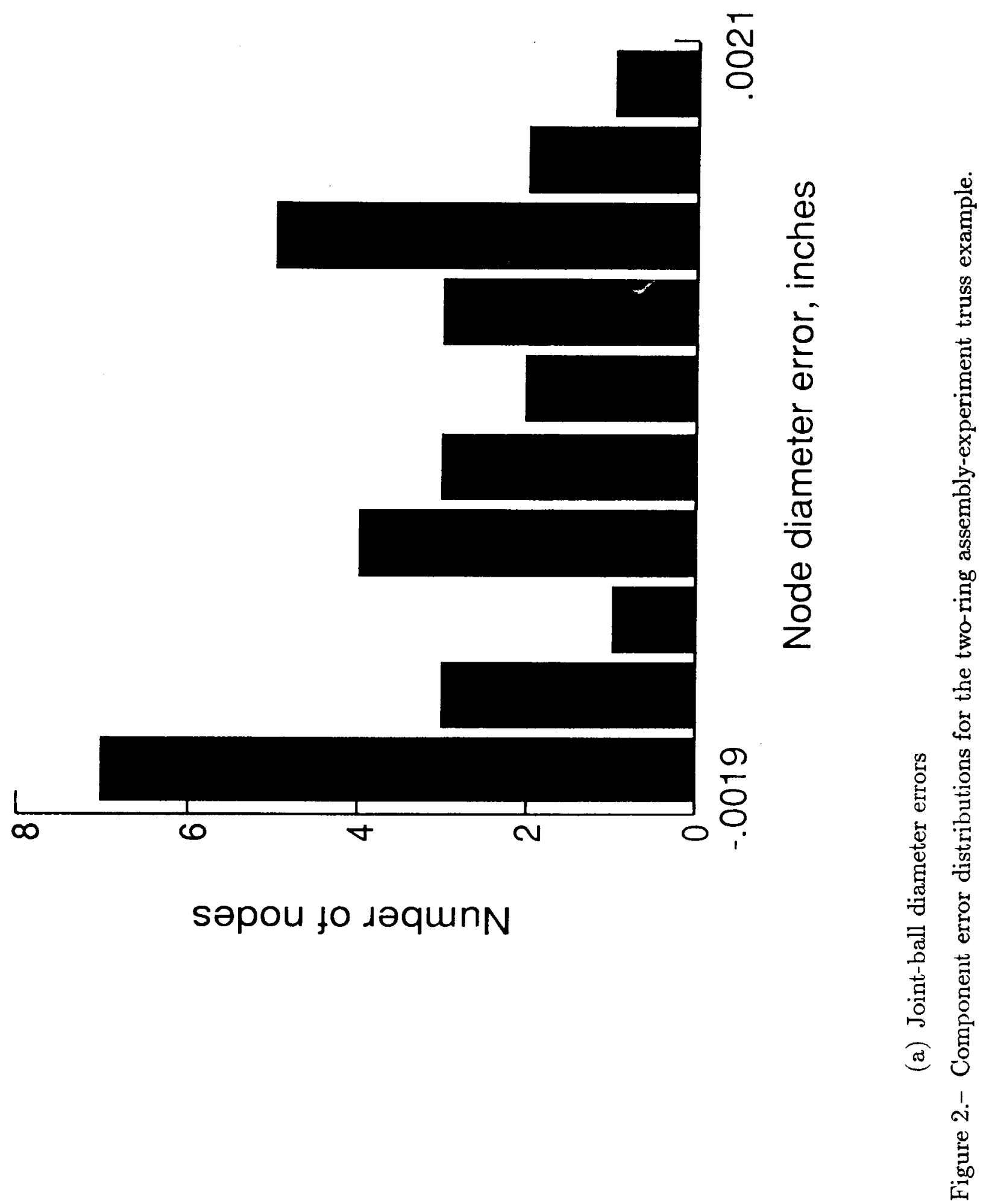


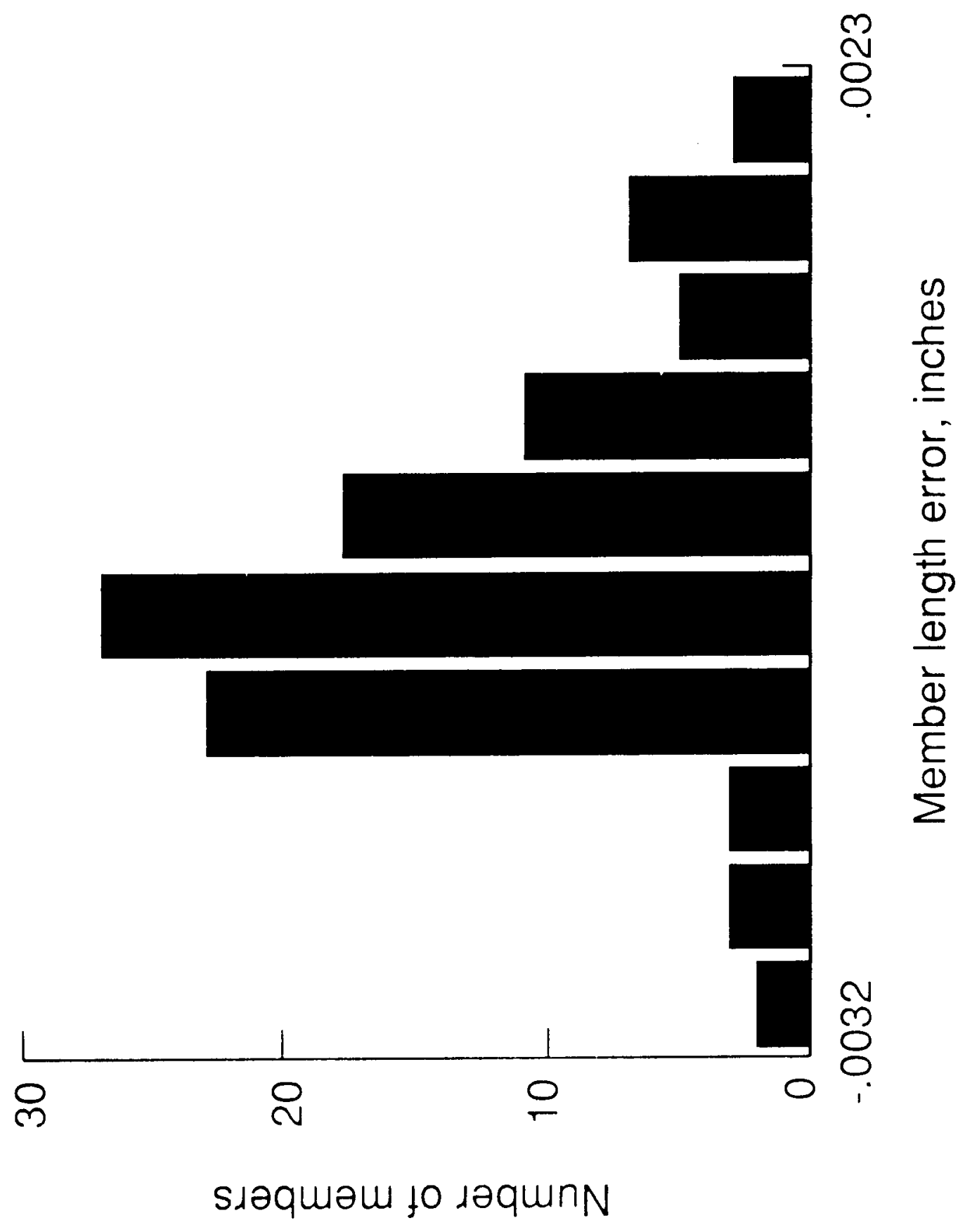

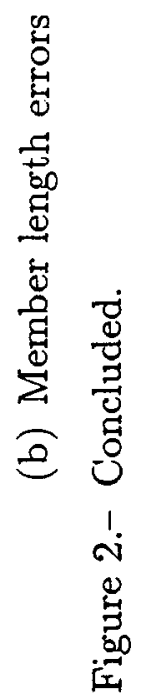




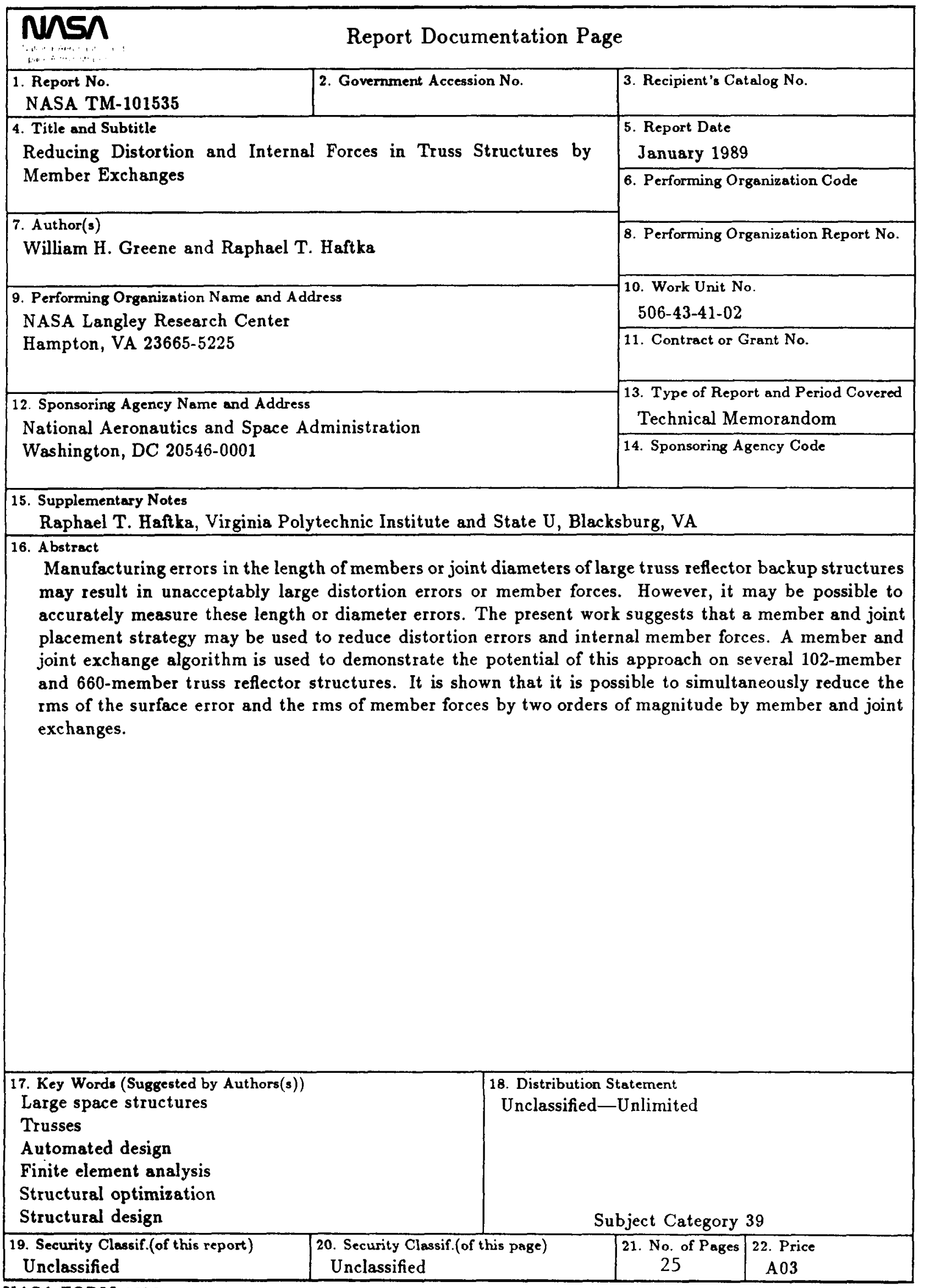

